\title{
A Novel Strategy for Preventing and Treating Cancer: Alteration of Cancer-Associated Chromatin Configuration
}

\author{
Gao-De Li \\ Chinese Acupuncture Clinic, Liverpool, UK \\ Email: gaode_li@yahoo.co.uk
}

How to cite this paper: Li, G.-D. (2021) A Novel Strategy for Preventing and Treating Cancer: Alteration of Cancer-Associated Chromatin Configuration. Open Access Library Journal, 8: e7347

https://doi.org/10.4236/oalib.1107347

Received: March 24, 2021

Accepted: May 3, 2021

Published: May 6, 2021

Copyright $\odot 2021$ by author(s) and Open Access Library Inc.

This work is licensed under the Creative Commons Attribution International License (CC BY 4.0).

http://creativecommons.org/licenses/by/4.0/

\section{(c) (i) Open Access}

\begin{abstract}
The hallmarks of cancer are determined by cancer-associated gene expression pattern that is determined by cancer-associated chromatin configuration (CACC). Using non-carcinogenic genotoxic drugs (NCGDs) to alter CACC could change cancer-associated gene expression pattern, which in turn, might cause disappearance of some or whole hallmarks of cancer and as a result, cancer cells could be killed by either apoptosis or immune destruction. Therefore, cancer could be prevented and treated by administration of NCGDs that can alter CACC in cancer cells.
\end{abstract}

\section{Subject Areas}

Cell Biology, Molecular Biology, Oncology

\section{Keywords}

Cancer, Cancer-Associated Chromatin Configuration (CACC),

Non-Carcinogenic Genotoxic Drugs (NCGDs), Cancer Prevention,

Cancer Treatment

\section{Introduction}

Thirty-five years ago, we published a paper in which we first proposed that 3-dimensional (3D) genome architecture, or chromatin configuration, might be linked to gene activities and determine gene expression pattern in each cell type, and that abnormalities of chromatin configuration might cause cancer [1]. Growing evidence has strongly supported our hypothesis [2] [3] [4]. In our paper, we also first proposed that to beat cancer, more efforts should be made to turn cancer cell's abnormal chromatin configuration to normal. Once cancer 
cell's aberrant chromatin configuration becomes normal, the cellular features of cancer cells will disappear, which indicates a new field that diseases could be treated via alteration of 3D genome architectures in related cell types. Recently, we published a paper in which we proposed that cancer-associated 3D genome architecture could be defined as cancer-associated chromatin configuration (CACC), and using less-toxic drugs to alter CACC could make cancer cells become less harmful [5]. Interestingly, around two months after our paper was published, Russian scientists reported that curaxins can alter cancer cell's genome architecture, which leads to suppression of many oncogenes [6].

Since the outbreak of the COVID-19 pandemic, repurposing old drugs to fight SARS-CoV-2 which causes COVID-19 has become a hot research topic. Unlike mainstream's virus-killing strategy, we proposed that non-carcinogenic genotoxic drugs (NCGDs) could be used to reduce target cell's susceptibility to SARS-CoV-2 and to modulate immune cell's response to SARS-CoV-2 infections through altering 3D genome architectures in target cells and immune cells. We also emphasized that altering 3D genome structures by NCGDs could be a cure-all strategy for preventing and treating all viral infections and many other diseases that are linked to aberrant 3D genome structures in related cells. Since cancer remains a leading cause of death worldwide, in this paper, we would like to explore in depth the effects of altering $3 \mathrm{D}$ genome architecture in carcinogenesis and de-cancerization so that a good strategy for preventing and treating cancer could be figured out.

\section{Linkage of Alteration of 3D Genome Architecture to both Carcinogenesis and De-Cancerization}

Based on our hypothesis, chromatin configuration, i.e., 3D genome architecture, determines cell type and different cell types contain different chromatin configurations [1]. Therefore, it is reasonable to assume that normal cell becoming cancer cell, i.e., carcinogenesis and cancer cell becoming normal or less harmful cell, i.e., de-cancerization, are undoubtedly associated with alterations of chromatin configurations. The driving force for these alterations is genotoxic agents that can alter chromatin configuration through various approaches, such as DNA damage, gene mutations, non-coding DNA mutations, non-DNA-damage intercalation, and epigenetic changes, such as DNA methylation landscape change etc. The detailed description of carcinogenesis and de-cancerization is presented as follows.

Cancer initiation is due to lone-term exposure to carcinogenic genotoxic agents that can abnormalize normal cell's chromatin configuration. During this process, most cells with aberrant chromatin configurations will die because the aberrant chromatin configurations are usually fatal. Only a small number of cells with CACC will survive and these cells will eventually develop into clinically detectable cancer. This process could be named as genotoxic agent-induced carcinogenesis (Figure 1(a)). Cancer cell is characterized by uncontrollable growth, 


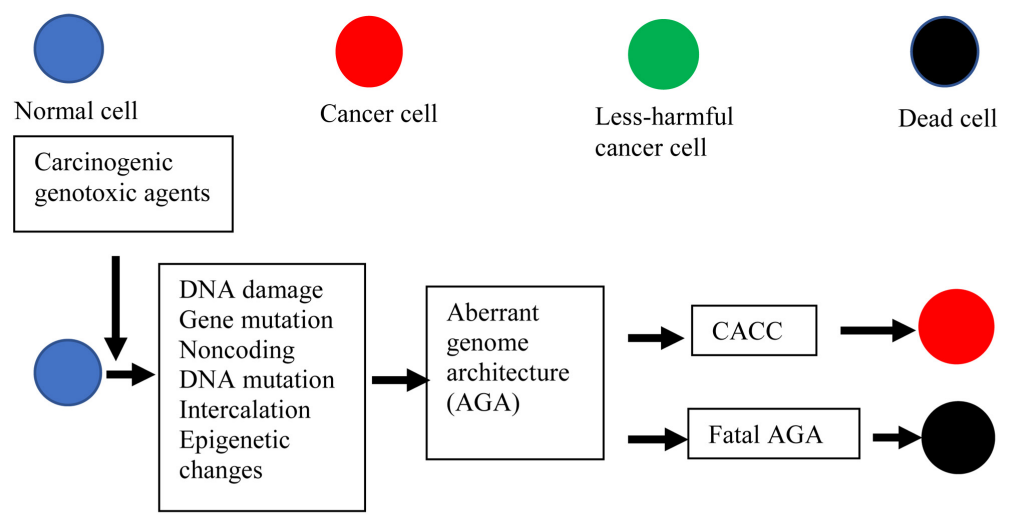

(a)

Non-carcinogenic
genotoxic agents

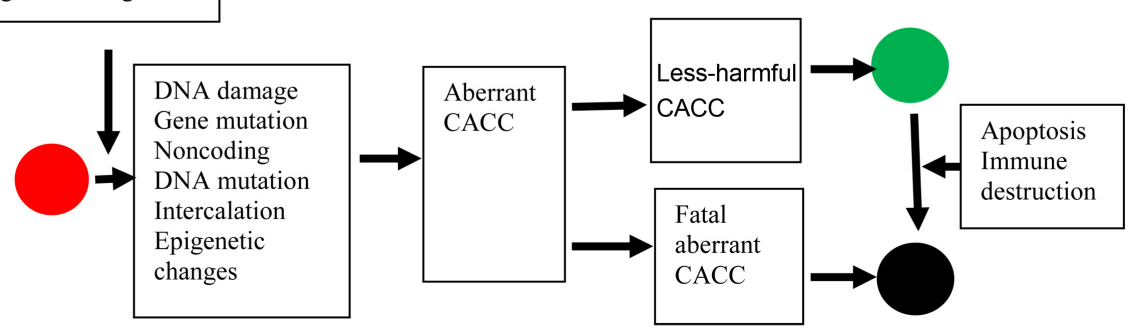

(b)

Figure 1. The schematic diagram shows how genotoxic agents cause and cure cancer through altering genome architectures. (a) Genotoxic agent-induced carcinogenesis; (b) Genotoxic agent-induced de-cancerization.

metastasis, avoiding apoptosis and immune destruction etc., which are named as the hallmarks of cancer [7]. It is due to these hallmarks that cancer causes death.

Recently, we proposed that NCGDs could be used to prevent and treat cancer because genotoxic agents could alter CACC and thus, might alter cancer cell's gene expression pattern [8] [9]. Theoretically, if cancer cell's CACC is altered by NCGDs, cancer cell's gene expression pattern will be altered, which could make cancer cells become less-harmful cancer cells. We assume that once cancer cells become less-harmful cancer cells, some cancer hallmarks, such as spreading, avoiding apoptosis and immune destruction etc., might become less effective or completely disappear. It is also possible that NCGDs might alter 3D genome architectures in immune cells, which could make immune cells regain ability to kill cancer cells. The overall results are that cancer cells could be killed either by apoptosis or by immune destruction. This process could be named as genotoxic agent-induced de-cancerization (Figure 1(b)). Besides, differentiation therapy using all-trans-retinoic acid (ATRA) can cause cancer cells to turn into normal cells [10]. Since ATRA is a drug with genotoxic properties [11], we assume that the mechanism of differentiation therapy is to induce cancer cells into normal cells via alteration of CACC by NCGDs. Although genotoxic agents could be divided into two groups: carcinogenic genotoxic agents that cause cancer and NCGDs that might cure cancer, there is no clear line between them, for example, 
arsenic trioxide is a carcinogenic genotoxic agent, but when being properly used, it could function as one of NCGDs to treat cancer [12].

Spontaneous remission of cancer (SRC) is a very rare phenomenon [13], the mechanism of which is unknown. From our point of view [8], SRC could be linked to long-term exposure to some unknown genotoxic agents, which could be non-anticancer medicine with genotoxic side effects, or might be contained in foods, drinks, health supplements and environment. The unknown genotoxic agents could coincidentally alter CACC in the SRC patient's cancer type and 3D genome architectures in immune cells. We speculate that fever, viral infection, trauma and other pathological factors could synergistically enhance genotoxic agent's effects in alteration of genome architectures in both cancer cells and immune cells and thus, might trigger quick de-cancerization (Figure 1(b)).

Taken together, cancer is caused by genotoxic agents and might be cured by genotoxic agents, which agrees with a wise Chinese saying that the person who tied the bell on tiger's neck is the person who is capable of taking it off.

\section{Repurposing Old Drugs with Genotoxic Side Effects as NCGDs for Cancer Prevention and Treatment}

NCGDs are new conceptual drugs that could be used to treat cancer and many other diseases [9]. The mechanism by which NCGDs exert their actions is changing gene expression pattern through altering 3D genome architectures in related cell types including cancer-cell types. One single of NCGDs usually can alter genome architectures in many cell types and thus could be used for treating a wide spectrum of diseases, the phenomenon of which could be named as "the cure-all properties" of NCGDs.

Currently, no NCGDs that are designed to alter 3D genome architecture are available in clinical practice. However, it is possible to repurpose some old drugs as NCGDs. The guiding principles for picking out NCGDs from old drugs are: first, the old drugs must be safe, and will not cause cancer in humans when being properly used; second, the old drugs must have genotoxic side effects that are proved by genotoxicity testing [14]. We speculate that drugs with genotoxic side effects might be able to alter 3D genome architectures in many cell types including normal cell types and cancer-cell types. To test this speculation, related experiments and clinical trials should be done. Based on these guiding principles, many old drugs with genotoxic side effects but without causing cancer in humans after long-term use could be picked out from nearly all drug classes, which could be repurposed as NCGDs for preventing and treating cancer. Interestingly, many such drugs have been proved to have more or less anticancer effects, such as vitamin C [15], aspirin [16], paracetamol [17], chloroquine [18], metformin [19], curcumin [20] and berberine [21] etc. The mechanisms by which these drugs exert their anticancer actions are various according to mainstream views, but we think that possessing genotoxic side effects is a common feature of these drugs, which might directly or indirectly alter CACC in related cancer types. 
Collectively, repurposing old drugs with genotoxic side effects as NCGDs for preventing and treating cancer is possible and worthy of systematic investigations. Since cancer usually grows undetected for about 10 years in humans, it is better to periodically take a long course of treatment with NCGDs once every 3 to 5 years, which could kill existing small number of cancer cells and thus, prevent cancer formation.

\section{Conclusions}

Carcinogens cause gene mutations and accumulated gene mutations cause normal cells to turn into cancer cells, which has long been common knowledge about carcinogenesis. Based on this knowledge, it is impossible to prevent cancer by administration of drugs because no drugs can be used to prevent or correct gene mutations in cancer cells.

Thirty-five years ago, we proposed a hypothesis that carcinogenesis might belinked to abnormalities of 3D genome architecture and as such, treating cancer should focus on alteration of cancer's abnormal 3D genome architecture [1], which opens up a new perspective in cancer management. Based on our hypothesis, gene mutations will not directly cause cancer. Normal cells becoming cancer cells is a cell type change, which is directly linked to alteration of 3D genome architecture. Therefore, to prevent and treat cancer, we can ignore gene mutations, and just pay attention to alteration of cancer-associated genome architecture or CACC. Genotoxic drugs are only drugs that can be used to alter CACC, which is why we proposed that NCGDs could be used for the prevention and treatment of cancer. Since no NCGDs are available currently, repurposing some old drugs with genotoxic side effects as NCGDs for these purposes is possible.

The advent of antibiotics is one of the greatest advances in therapeutic medicine. Thanks to antibiotics, the number of deaths from bacterial infections has dramatically reduced. However, there are many diseases that are not caused by bacteria but by abnormalities of gene expression patterns in related cell types, such as aging-associated diseases. To treat these abnormal gene expression pattern-associated diseases, manipulating gene expression patterns through altering 3D genome architectures in related cell types by NCGDs might be a promising strategy.

\section{Conflicts of Interest}

The author declares no conflicts of interest regarding the publication of this paper.

\section{References}

[1] Li, G.D. (1986) Abnormal Chromatin Configuration and Oncogenesis. Medicine and Philosophy, 7, 12-14. (In Chinese)

[2] Corces, M.R. and Corces, V.G. (2016) The Three-Dimensional Cancer Genome. 
Current Opinion in Genetics \& Development, 36, 1-7.

https://doi.org/10.1016/j.gde.2016.01.002

[3] Flavahan, W.A., Drier, Y., Liau, B.B., Gillespie, S.M., Venteicher, A.S., Suva, M.L., Stem-Mer-Rachamimov, A.O. and Bernstein, B.E. (2016) Insulator Dysfunction and Oncogene Activation in IDH Mutant Gliomas. Nature, 529, 110-114.

https://doi.org/10.1038/nature16490

[4] Feng, Y. and Pauklin, S. (2020) Revisiting 3D Chromatin Architecture in Cancer Development and Progression. Nucleic Acids Research, 48, 10632-10647. https://doi.org/10.1093/nar/gkaa747

[5] Li, G.D. (2019) Further Thoughts on Abnormal Chromatin Configuration and Oncogenesis. Open Access Library Journal, 6, e5185.

https://doi.org/10.4236/oalib.1105185

[6] Kantidze, O.L., Luzhin, A.V., Nizovtseva, E.V., Safina, A., Valieva, M.E. and Golov,A.K., et al. (2019) The Anti-Cancer Drugs Curaxins Target Spatial Genome Organization. Nature Communications, 10, Article No. 1441. https://doi.org/10.1038/s41467-019-09500-7

[7] Hanahan, D. and Weinberg, R.A. (2000) The Hallmarks of Cancer. Cell, 100, 57-70. https://doi.org/10.1016/S0092-8674(00)81683-9

[8] Li, G.D. (2020) Targeting Three-Dimensional Genome Architecture Might Be One of the Mechanisms of Chloroquine's Diverse Therapeutic Actions. Open Access Library Journal, 7, e6340. http://doi.org/10.4236/oalib.1106340

[9] Li, G.D. (2020) Non-Carcinogenic Genotoxic Drugs Could Be Used to Prevent and Treat COVID-19. Open Access Library Journal, 7, e6536. https://doi.org/10.4236/oalib.1106536

[10] Yan, M. and Liu, Q. (2016) Differentiation Therapy: A Promising Strategy for Cancer Treatment. Chinese Journal of Cancer, 35, Article No. 3. https://doi.org/10.1186/s40880-015-0059-x

[11] Alakhras, RS., Stephanou, G., Demopoulos, N.A. and Nikolaropoulos, S.S. (2011) Genotoxicity of All-Trans Retinoic Acid (ATRA) and Its Steroidal Analogue EA-4 in Human Lymphocytes and Mouse Cells in Vitro. Cancer Letters, 306, 15-26. https://doi.org/10.1016/j.canlet.2011.02.010

[12] Miller Jr., W.H., Schipper, H.M., Lee, J.S., Singer, J. and Waxman, S. (2002) Mechanisms of Action of Arsenic Trioxide. Cancer Research, 62, 3893-903.

[13] Cole, W.H. and Everson, T.C. (1956) Spontaneous Regression of Cancer: Preliminary Report. Annals of Surgery, 144, 366-383. https://doi.org/10.1097/00000658-195609000-00007

[14] Turkez. H., Arslan, M.E. and Ozdemir, O. (2017) Genotoxicity Testing: Progress and Prospects for the Next Decade. Expert Opinion on Drug Metabolism \& Toxicology, 13, 1089-1098. https://doi.org/10.1080/17425255.2017.1375097

[15] Butt, G., Farooqi, A.A., Adylova, A., Attar, R., Yilmaz, S., Konysbayevna, K.K., Sabitaliyevich, U.Y., Gasparri, M.L. and Xu, B. (2020) Vitamin C as an Anticancer Agent: Regulation of Signaling Pathways. Current Topics in Medicinal Chemistry, 20, 1868-1875. https://doi.org/10.2174/1568026620666200710102841

[16] Song, Y., Zhong, X., Gao, P., Zhou, C., Shi, J., Wu Z., Guo, Z. and Wang, Z. (2020) Aspirin and Its Potential Preventive Role in Cancer: An Umbrella Review. Frontiers in Endocrinology, 11, Article No. 3. https://doi.org/10.3389/fendo.2020.00003

[17] Bonovas, S., Filioussi, K. and Sitaras, N.M. (2006) Paracetamol Use and Risk of Ovarian Cancer: A Meta-Analysis. British Journal of Clinical Pharmacology, 62, 
113-121. https://doi.org/10.1111/j.1365-2125.2005.02526.x

[18] Zhang, Y., Liao, Z., Zhang, L.J. and Xiao, H.T. (2015) The Utility of Chloroquine in Cancer Therapy. Current Medical Research and Opinion, 31, 1009-1013. https://doi.org/10.1185/03007995.2015.1025731

[19] Vancura, A., Bu, P., Bhagwat, M., Zeng, J. and Vancurova, I. (2018) Metformin as an Anticancer Agent. Trends in Pharmacological Sciences, 39, 867-878. https://doi.org/10.1016/j.tips.2018.07.006

[20] Tomeh, M.A., Hadianamrei, R. and Zhao, X. (2019) A Review of Curcumin and Its Derivatives as Anticancer Agents. International Journal of Molecular Sciences, 20, Article No. 1033. https://doi.org/10.3390/ijms20051033

[21] Wang, Y., Liu, Y., Du, X., Ma, H. and Yao, J. (2020) The Anti-Cancer Mechanisms of Berberine: A Review. Cancer Management and Research, 12, 695-702. https://doi.org/10.2147/CMAR.S242329 